\title{
ANÁLISE DOS IMPACTOS DO USO DO SOLO SOBRE A DIVERSIDADE DE BESOUROS ROLA-BOSTA NA REGIÃO DO CAPARAÓ CAPIXABA
}

\author{
Sabrina Buqueroni Alves ${ }^{1}$ \\ Andressa Hartuiq dos Santos ${ }^{2}$ \\ Keminy Ribett Bautz ${ }^{3}$ \\ Mariana Almeida dos Santos ${ }^{4}$ \\ Pedro Henrique Charpinel Giestas ${ }^{5}$ \\ Ronara de Souza Ferreira ${ }^{6}$
}

Resumo: A modificação do ambiente nativo proveniente de atividades antrópicas, como a agropecuária, tem transformado grandes áreas de vegetação contínua em um mosaico de áreas modificadas, interferindo assim em toda fauna existente no local. O presente trabalho teve como objetivo analisar como os diferentes sistemas de uso do solo (SUS) na região do Caparaó Capixaba, sul do Espírito Santo, afetam a biodiversidade das comunidades de besouros escarabeíneos, modificando sua riqueza, abundância, composição e biomassa. Foram amostradas 10 áreas contendo 3 pontos amostrais em cada, para cada SUS (fragmentos florestais, cafezais e pastagens), totalizando um esforço amostral de 90 pontos amostrais. Foram coletados 1.063 indivíduos de 49 espécies diferentes. Ao analisar os resultados obtidos observamos algumas diferenças na riqueza, abundância, composição e biomassa entre os SUS estudados, provando que a diminuição da complexidade ambiental através de atividades antrópicas, acarreta na perda da biodiversidade desses insetos tão importantes para o funcionamento dos ecossistemas.

Palavras-chave: Modificação ambiental; Uso do solo; Caparaó capixaba; Besouros rola-bosta.

\footnotetext{
${ }^{1}$ Universidade Federal do Espírito Santo, Centro de Ciências Exatas, Naturais e da Saúde, Departamento de Biologia, Brasil. E-mail: sabrinabuqueroni21@gmail.com.

2 Universidade Federal do Espírito Santo, Centro de Ciências Exatas, Naturais e da Saúde, Departamento de Biologia, Brasil. E-mail: andressahartuiq@yahoo.com.br.

3 Universidade Federal do Espírito Santo, Centro de Ciências Exatas, Naturais e da Saúde, Departamento de Biologia, Brasil. E-mail: keminy.ribet@hotmail.com.

4 Universidade Federal do Espírito Santo, Centro de Ciências Exatas, Naturais e da Saúde, Departamento de Biologia, Brasil. E-mail: mari.almeida.s@hotmail.com.

5 Universidade Federal do Espírito Santo, Centro de Ciências Exatas, Naturais e da Saúde, Departamento de Biologia, Brasil. E-mail: pedrin_giestas@hotmail.com.

6 Universidade Federal do Espírito Santo, Centro de Ciências Exatas, Naturais e da Saúde, Departamento de Biologia, Brasil. E-mail: ronara.ferreira@gmail.com.
} 